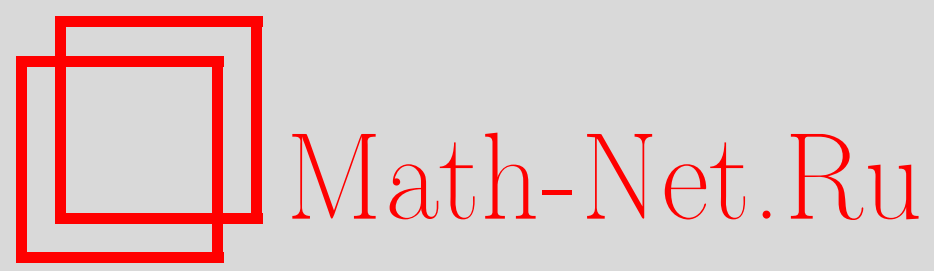

Ю. И. Кольцун, Механика концентрации напряжений в окрестности фронта нераспространяющейся усталостной трещины, Вестн. Сам. гос. техн. ун-та. Сер. Физ.-мат. науки, 2004, выпуск 30, 41-54

DOI: https://doi.org/10.14498/vsgtu306

Использование Общероссийского математического портала Math-Net.Ru подразумевает, что вы прочитали и согласны с пользовательским соглашением

http://www.mathnet.ru/rus/agreement

Параметры загрузки:

IP : 107.22 .136 .117

26 апреля 2023 г., 06:37:06 


\title{
Ю.И. Кольцун
}

\section{МЕХАНИКА КОНЦЕНТРАЦИИ НАПРЯЖЕНИЙ В ОКРЕСТНОСТИ ФРОНТА НЕРАСПРОСТРАНЯЮЩЕЙСЯ УСТАЛОСТНОЙ ТРЕЩИНЫ}

\begin{abstract}
На основе реальной нераспространяющейся усталостной трещины серповидной формы в соответствии с линейной механикой разрушения проведен анализ конщентрации номинальных напряжений в окрестности ее фронта при изгибе иилиндрической детали из средне углеродистой стали с кольцевым надрезом. Введено понятие локального теоретического коэффициента концентращии напряжений на дне трещины. Предложены две законообразующие формуль, определяющие связь концентрации макронапряжений на дне конструктивного концентратора и на дне усталостной трещины, а также связь теоретического коэффициента концентрации напряжений детали и локального теоретического коэффициента концентрации напряжений на дне трещины с расстоянием между двумя симметричными относительно нейтральной оси серповидными нераспространяюшимися усталостными трещинами при изгибе детали. Выведена формула максимальной глубины серповидной усталостной макротрещиныл.
\end{abstract}

Современные представления о кинетике трещины усталости в инкубационный период, от момента ее страгивания до достижения критической глубины или длины, а также на этапе развития магистральной трещины базируются на экспериментально-теоретических исследованиях зарубежных ученых А.А. Гриффитса, Дж.Р. Ирвина, Е.О.Орована, Дж.Р.Райса, Д.Дагдейла, А.Т.Екобори.. В России проблемами механики разрушения занимались и занимаются в настоящее время такие крупные ученые как И.В.Обреимов, Г.П.Черепанов, П.И.Кудрявцев, Е.М.Морозов, В.З.Партон и многие другие [1-18]. В качестве образцов для исследований в основном использовался и используется тонкий листовой материал или тонкие цилиндрические стержни из различных материалов, при этом в исследуемой зоне внешней нагрузкой создается либо линейное напряженное состояние /ЛНС/ (центральное растяжение), либо плоское напряженное состояние /ПНС/ (кручение, изгиб, двухосное растяжение). Исследованию процессов развития усталостных трещин в условиях концентрации напряжений при плоской деформации и в деталях со значительными размерами поперечного сечения посвящено меньшее количество работ в силу чисто объективных обстоятельств, связанных и с наличием сложного испытательного оборудования, и с изготовлением образцов, и с большими экономическими затратами. В Советской России на образцах, идентичных реальным деталям, широкие научные исследования с целью определения предела выносливости по первой макротрещине при изгибе, кручении, растяжении-сжатии проводили И.В.Кудрявцев и П.И.Кудрявцев [6,7].

Усталостные изломы разрушенных деталей и образцов с трещинами в основном подвергаются металлографическому анализу с использованием оптических и электронных микроскопов. Однако тщательный физико-математический анализ статических изломов деталей с наличием в них усталостных трещин практически не проводился. В то время как исследование геометрических закономерностей развития усталостных трещин в сечении излома непосредственно связано не только с развитием деформаций и движением дислокаций, но и с изменением величины действительных макронапряжений.

Целью настоящей публикации является постулирование нового направления исследований в области механики разрушения - физико-математический анализ геометрии излома детали с усталостной трещиной, представление методики и результатов анализа.

В качестве примера используется статический излом при изгибе цилиндрической детали из среднеуглеродистой стали 45 диаметром 25 мм с кольцевым надрезом полукруглого профиля радиусом 1мм, нанесенным безнаклепным способом на предварительно упрочненную поверхность обкаткой роликом с усилием 1 кН , выстоявшей 10 млн. циклов при симметричном цикле на предельном уровне амплитудных напряжений 192 МПа, близком к пределу выносливости детали, равном 194МПа.Частота испытаний 19,5Гц , среднее квадратичное отклонение предела выносливости по разрушению составляет 13,6 МПа.

На рис. 1 изображена фотография анализируемого излома. Предварительным основанием для физико-математического анализа геометрии излома является разделение на зоны, соответствующие нераспространяющейся усталостной трещине 1 , долома 2 , области охрупчивания 3 , магистральной трещине 4. Усталостная трещина 1 имеет темный фон, а область охрупчивания 3 - светлый. Видно, что область охрупчивания по площади несколько превышает площадь нераспространяющейся усталостной трещины. Графическое моделирование аналога исследуемо- 
го поперечного сечения показывает, что центральный угол, полностью охватывающий серповидную трещину, составляет строго $120^{\circ}$. Со стороны, противоположной усталостной трещине, в области магистральной трещины, вблизи поверхности дна кольцевого надреза также прорисовывается нераспространяющаяся усталостная трещина, имеющая выпуклую площадку в пределах центрального угла, равного $60^{\circ}$.

На рис. 2 изображен излом аналогичной детали при амплитуде напряжений, в полтора раза превышающей предел выносливости по разрушению, т.е. при 288 МПа. Так как разрушение во втором случае произошло через 35000 циклов, более темная область соответствует максимальной плотности дислокаций. Данная область характеризуется наличием микротрещин под поверхностью в зоне, непосредственно прилегающей к силовой плоскости. Отчетливо виден чечевицеобразный наплыв в середине серповидной области, определяющий окончательную форму предстоящей усталостной трещины. Данная практически плоская форма усталостной трещины, которая затем переходит в магистральную, характеризующуюся морщинистыми линиями усталостных бороздок, видна в нижней части и на рис.1.

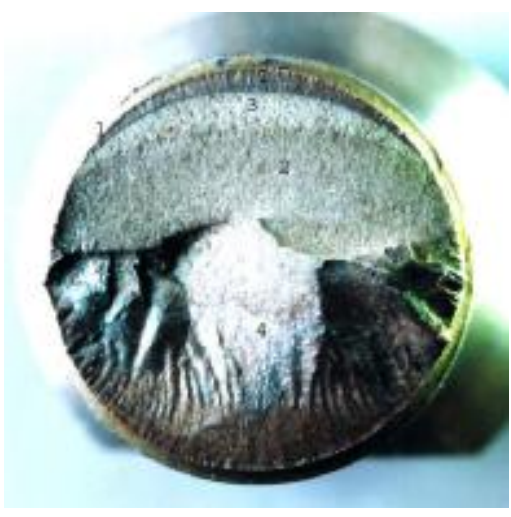

Р и с. 1. Статический излом детали из стали 45 с полукруглым надрезом $r=1$ мм, выстоявшей базу испытаний $\mathrm{N}=10$ млн.циклов при изгибе

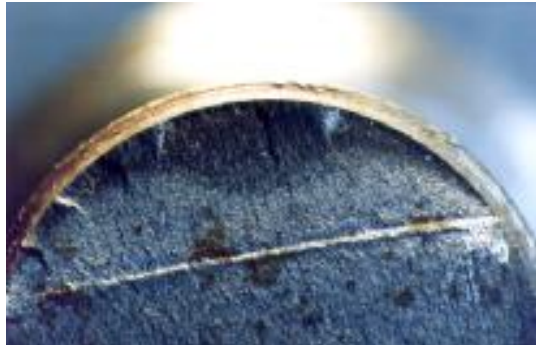

Р и с. 2. Усталостный излом исследуемой детали из стали 45 с превышением предельной амплитуды в 1.5 раза

Учитывая тот факт, что наплыв определяет переход от нераспространяющейся усталостной трещины к магистральной, то, естественно, возникает первый вопрос: какова разница в величинах действительных напряжений $\sigma^{\text {действ }}$ на поверхности дна надреза в пределах центрального угла $\Theta$ от силовой плоскости, т.е. от $0^{\circ}$ до $\Theta_{э \phi}$, определяемого половину максимальной длины наплыва?

Испытания, направленные на регистрацию усталостной трещины с использованием стробоскопа СШ2 и 15-кратного микроскопа свидетельствуют о том, что в пределах двойного центрального угла $\Theta_{э ф}$ усталостная трещина растет очень медленно, При достижении длины трещины на поверхности дна надреза в опасном сечении, соответствующей длине дуги окружности в пределах $2 \Theta_{э \phi}$, дальнейший ее рост происходит практически мгновенно с остановкой в пределах двойного центрального угла $\Theta_{\max }$, что определяет второй вопрос: почему скорость увеличения длины усталостной трещины по поверхности дна надреза в опасном сечении в пределах от $\Theta_{э \phi}$ до $\Theta_{\max }$ несопоставимо больше скорости в пределах центрального угла от $0^{\circ}$ до $\Theta_{э ф}$ ?

Третий вопрос: возможно ли определить предел выносливости детали по образованию первой макротрещины, исходя из геометрии усталостного излома детали с трещиной?

Четвертый вопрос: какова максимальная глубина усталостной трещины серповидной формы, гарантирующая безопасную эксплуатацию детали с трещиной, работающей при изгибe?

Ответы на эти вопросы определяют методику физико-математического анализа геометрии излома детали с нераспространяющейся усталостной трещиной, а решение четвертого вопроса определяет критерий безопасного повреждения детали. Отдельные исследования по этим вопросам опубликованы автором в работах [2-5]. 


\section{1. Физический анализ}

1.1. Предел выносливости детали по первой макротрещине. На рис.3 изображено опасное сечение после разрыва образца с указанием границ фронтов серповидных нераспространяющихся трещин усталости, выделенных дугами, проходящими через точки В. Положения точек В определяют места остановки движения трещины в силу уменьшения действи-

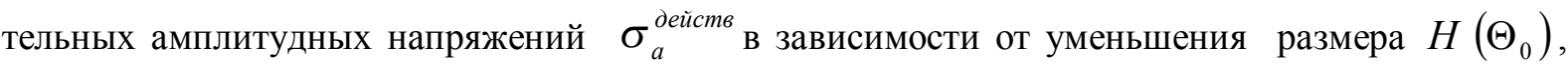
эквивалентного текущей координате у в формуле для номинальных амплитудных напряжений $\sigma_{a}$ от $d / 2$ (точка А) до размера $H\left(\Theta_{\max }\right)$ в точке В. Здесь $\Theta-$ угловая координата движения фронта трещины по поверхности опасного сечения цилиндрического образца;

$$
\sigma_{a}=\frac{M_{\text {uз2 }}}{I_{x}} \cdot y,
$$

где $\mathrm{M}_{\text {изг }}$ - изгибающий момент в силовой плоскости, $I_{x}$ - момент инерции опасного сечения.

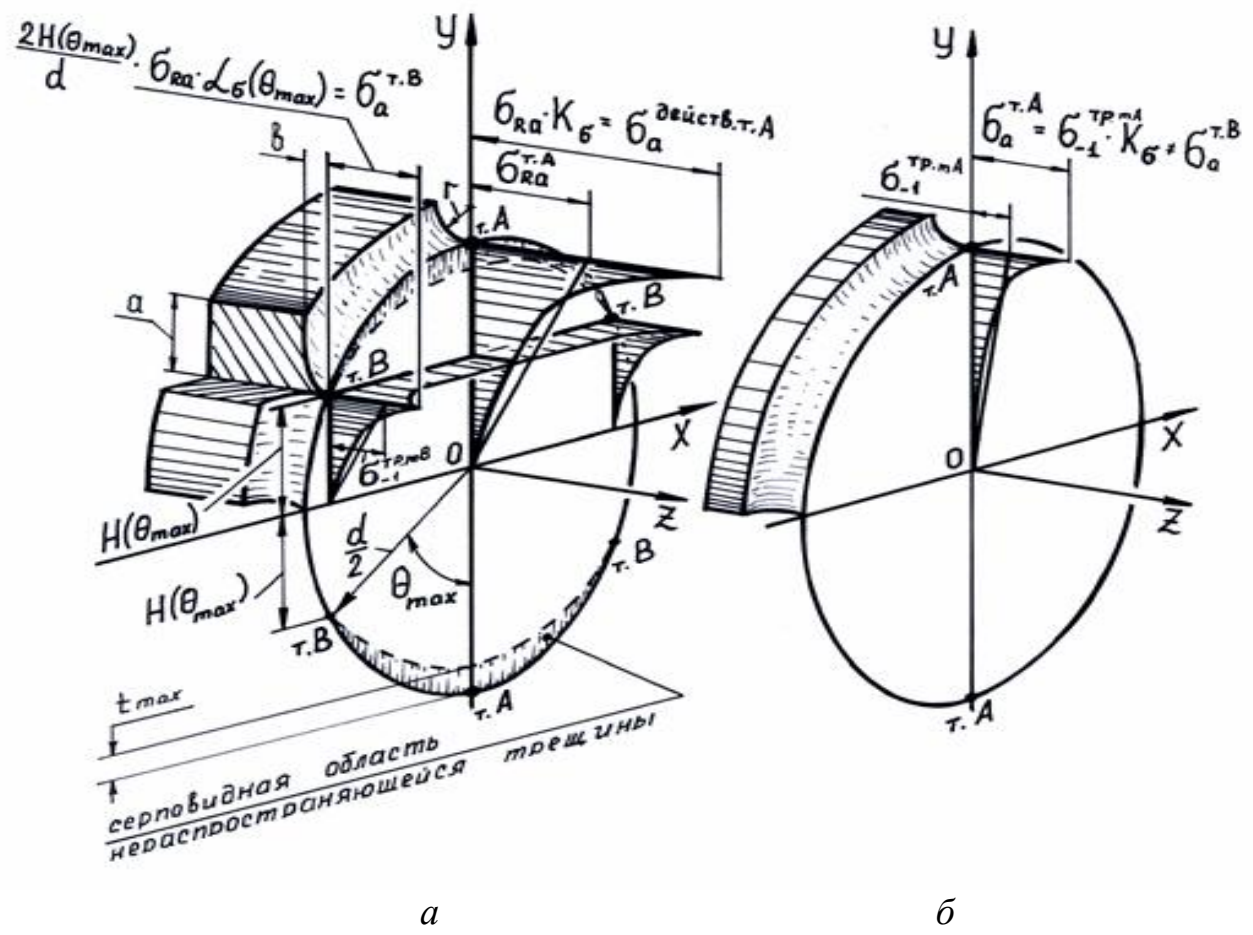

Р и с. 3. Распределение действительных напряжений в опасном сечении детали при изгибе

Исходя из равенства отношения номинальных напряжений $\frac{\sigma_{R a}^{T . A}}{\sigma_{a}^{T . B}}=\frac{d / 2}{H\left(\Theta_{\max }\right)}$, следует:

$$
\sigma_{a}^{T . B}=\frac{2 H\left(\Theta_{\max }\right)}{d} \cdot \sigma_{R a}^{T . A} \cdot
$$

В то же время действительные амплитудные напряжения в точке В в силу концентрации напряжений равны:

$$
\sigma_{a}^{\text {действ.T.B }}=\sigma_{a}^{T . B} \cdot \alpha_{\sigma}\left(\Theta_{\max }\right)=. \frac{2 H\left(\Theta_{\max }\right)}{d} \cdot \sigma_{R a}^{T . A} \cdot \alpha_{\sigma}\left(\Theta_{\max }\right) .
$$

С другой стороны, при незначительном превышении номинальных амплитудных напряжений предельной величины $\sigma_{R a}$ произойдет разрушение образца. В этом случае действительные напряжения в точке $\mathrm{B}$, которые будут являться разрушающими для цилиндрической детали с внутренним диаметром $2 \cdot \mathrm{H}\left(\Theta_{\max }\right)$ и полуэллиптическим надрезом, соответствующим вертикальному сечению кольцевого полукруглого надреза детали в точке $\mathrm{B}$, параллельному силовой плоскости, можно представить равными произведению эффективного коэффициента концентрации напряжений $\mathrm{K}_{\sigma}\left(\alpha_{\sigma}\left(\Theta_{\max }\right)\right)=\mathrm{K}_{\sigma}\left(\Theta_{\max }\right)$ и $\sigma_{a}^{T . B}$. Тогда номинальные амплитудные на- 
пряжения в точке В $\sigma_{a}^{T . B}$ можно переобозначить в предел выносливости детали, т.е. образца с концентратором, по образованию первой макротрещины в точке В $-\sigma_{-1 \text { деm }}^{\text {TPm.B }}$ :

$$
\sigma_{-1 \partial e m}^{T P m . B}=\frac{2 \cdot \mathrm{H}\left(\Theta_{\max }\right)}{d} \cdot \sigma_{R a} \cdot \frac{\alpha_{\sigma}\left(\Theta_{\max }\right)}{\mathrm{K}_{\sigma}\left(\Theta_{\max }\right)} .
$$

Так как эффективный коэффициент концентрации напряжений представляет собой отношение предела выносливости образца без концентратора, т.е. образца гладкого, к пределу выносливости образца с концентратором, т.е. детали, и является безразмерной относительной величиной определяющей физические параметры разрушения, то в первом приближении реально предположить, что отношение предела выносливости по первой макротрещине образца без концентратора к пределу выносливости по первой макротрещине с концентратором можно также выразить через эффективный коэффициент концентрации напряжений. В этом случае для точки В получаем следующее соотношение:

$$
\sigma_{-1 \text { гладк }}^{T P \text {. }}=\sigma_{-1 \text { деm }}^{T P m} \cdot \mathrm{K}_{\sigma}\left(\Theta_{\max }\right) .
$$

При переходе к гладкой детали с другим диаметром, определяемым, например, точкой А, в окрестности которой экспериментально регистрируется трещина, целесообразно учитывать изменение линейных размеров опасного сечения путем использования коэффициента влияния абсолютного размера $\mathrm{K}_{d}$, функционально зависящего от линейного размера $2 \mathrm{H}\left(\Theta_{\max }\right)$ в точке $\mathrm{B}$, и от внутреннего диаметра опасного сечения $d$ в точке А. В этом случае:

$$
\frac{\sigma_{-1 \text { dem }}^{T P . m . B}}{\mathrm{~K}_{d}\left(2 \mathrm{H}\left(\Theta_{\max }\right)\right)}=\frac{\sigma_{-1 \text { dem }}^{T P . m . A}}{\mathrm{~K}_{d}(d)} \cdot \mathrm{K}_{\sigma}(d) .
$$

Подставляя выражение (4) в (6) и выделяя предел выносливости детали по первой макротрещине в точке А, получаем следующее выражение:

$$
\sigma_{-1 \text { dem }}^{T P m . A}=\frac{2 H\left(\Theta_{\max }\right)}{d} \cdot \sigma_{R a}^{T . A} \cdot \alpha_{\sigma}\left(\Theta_{\max }\right) \cdot \frac{1}{\mathrm{~K}_{\sigma}(d) \cdot \mathrm{K}_{\sigma}\left(\Theta_{\max }\right)} \cdot \frac{\mathrm{K}_{d}(d)}{\mathrm{K}_{d}\left(2 H\left(\Theta_{\max }\right)\right.} \cdot
$$

В выражении (7) теоретическому коэффициенту концентрации напряжений $\alpha_{\sigma}\left(2 H\left(\Theta_{\max }\right)\right)$ целесообразно дать определение локальный, который можно дифференцированно определять путем использования прочностных программ ANSYS или MNS NASTRAN. Эффективный коэффициент концентрации напряжений определяется по известной формуле [13]

$$
\mathrm{K}_{\sigma}=1+q_{\sigma} \cdot\left(\alpha_{\sigma}-1\right),
$$

где $q_{\sigma}$ - коэффициент чувствительности к концентрации напряжений.

В силу изменения формы концентратора в секущей вертикальной плоскости при изменении текущего угла $\Theta$ (рис.1, а) концентрация напряжений по контуру сечения должна увеличиваться. Учитывая вышеизложенное, расчет теоретического коэффициента концентрации напряжений в силовой плоскости можно выполнять по формуле Нейбера [10] для полуэллиптического концентратора:

$$
\alpha_{\sigma} \cong 1+2 \sqrt{\frac{a}{\rho}}=1+2 \cdot \frac{a}{b},
$$

где $b$ - малая полуось эллипса (половина ширины концентратора); $a$ - большая полуось эллипса (глубина концентратора),; $\rho$ - радиус кривизны эллипса на конце полуоси $a\left(\rho=b^{2} / a\right)$.

В случае изменения текущего центрального угла $\Theta$ глубина концентратора $b$ в вертикальной секущей плоскости, параллельной силовой плоскости $Y O Z$, определяется по следующей формуле:

$$
a=\frac{1}{2} \cdot\left(D \cdot \sqrt{1-\left(\frac{d}{D} \cdot \sin \Theta\right)^{2}}-d \cdot \cos \Theta\right),
$$

где $D$ - наибольший диаметр лабораторного образца; $d$ - диаметр опасного сечения образца. В соответствии с формулами (8)-(10) получаем : при $\Theta=0^{\circ}: \quad b \equiv r=1 \mathcal{M}, a=r=1$ м , $\alpha_{\sigma}(d)=3,0 \quad, \mathrm{~K}_{\sigma}=2,16$; при $\Theta \equiv \Theta_{\text {мах }}=60^{\circ}: b \equiv r=1$ мм $, \quad a=1,8$ мм $, \quad \alpha_{\sigma}\left(2 H\left(60^{\circ}\right)\right)=4,6$, $\mathrm{K}_{\sigma}=3,1$. 
На основании формулы (7) теперь можно вычислить предел выносливости по образованию первой макротрещины, зарождающейся в точке А исследуемого образца из стали 45 , в условиях концентрации напряжений, зная экспериментальное значение предельной амплитуды напряжений испытанной детали $\sigma_{R a}^{T \cdot A}=192 M П a$ :

$$
\sigma_{-1}^{T P . m . A}=\frac{2 \cdot 5,75}{23} \cdot 192 \cdot \frac{4,6}{2,16 \cdot 3,1} \cdot \frac{0,9}{0,97} \approx 61,0(\text { MПa }) .
$$

Сравнивая полученное значение с результатом исследования, проведенного П.И.Кудрявцевым в работе[6], где на графике зависимости предела выносливости по первой макротрещине от теоретического коэффициента концентрации напряжений для стали 45 при $\alpha_{\sigma}(d)=3,0$ $\sigma_{-1}^{T P}=65$ МПа, можно отметить вполне удовлетворительную сходимость.

Зная предел выносливости по образованию первой трещины, на основании кинетики трещины при напряжениях, превышающих предельную амплитуду в пределах эффективного центрального угла $\Theta_{э \phi}=\Theta_{\max } / 2$, можно определить допустимую амплитуду напряжений гарантированной безопасной работы детали с трещинами. Для этого целесообразно вычислить по формуле (7) напряжения при $\Theta_{э \phi}=60^{\circ} / 2=30^{\circ}$, которые соответственно равны 108 МПа.

В случае отсутствия концентратора напряжений, т.е. для гладкой рабочей зоны лабораторного образца, когда $\alpha_{\sigma}=1,0 ; \mathrm{K}_{\sigma}=1,0$, предел выносливости материала по образованию первой макротрещины для стали 45 равен

$$
\sigma_{-1}^{T P}=\frac{2 \cdot 5,75}{23} \cdot 192 \cdot \frac{1,0}{1,0 \cdot 1,0} \cdot \frac{0,9}{0,97} \cong 90(\text { МПа }) .
$$

Таким образом, используя предложенную методику постановки эксперимента, а также формулу (7) можно прогнозировать величину предела выносливости по образованию первой макротрещины для любых пластичных материалов, как в условиях концентрации напряжений, так и для гладкой рабочей зоны образца.

Исходя из вышеизложенного, следует заметить, что введено новое понятие - локальный теоретический коэффициент концентрации напряжений $\alpha_{\sigma}(\Theta)$, который требует более полного рассмотрения с точки зрения геометрического анализа опасного сечения рассматриваемой детали.

1.2. Локальный теоретический коэффициент концентрации напряжений. Особенностью, свидетельствующей о необходимости более подробного рассмотрения локальной концентрации напряжений, является тот факт, что распределение действительных нормальных напряжений по контуру дна кольцевого надреза в опасном сечении от нейтральной оси до точки А в некоторых пакетах прочностных программ рассчитывается по линейному закону. При увеличении глубины двух симметричных серповидных усталостных трещин, превышение одной из которых приводит к возникновению магистральной трещины, скорость движения фронтов трещин по поверхности дна надреза, т.е. скорость движения точки В зависит от теоретического коэффициента концентрации напряжений, определяемого изменением формы полукруглого надреза. Если положение точку В определяется угловой координатой $\Theta_{i}$, то при изгибе детали в секущих плоскостях, проходящих через точку В параллельно силовой плоскости, форма надреза будет представлять собой полуэллипс с большой полуосью $a$ и малой полуосью $b$, равной радиусу полукруглого надреза $\mathrm{r}$. С увеличением текущей угловой координаты $\Theta_{i}$ эллипсность надреза в секущей плоскости увеличивается и, значит, увеличивается и теоретический коэффициент концентрации напряжений $\alpha_{\sigma}\left(\Theta_{i}\right)$. Индекс $i$ означает использование возможности дискретного рассмотрения сечений через постоянное значение приращения центрального угла $\Theta$.

Возникает вопрос: возможно ли изменение теоретического коэффициента концентрации напряжений $\alpha_{\sigma}\left(\Theta_{i}\right)$ по линейному закону при движении точки В по контуру дна надреза? Расчет теоретического коэффициента концентрации напряжений по известной формуле Нейбера [10] для полуэллиптической формы концентраторов через $10^{\circ}$ по угловой координате $\Theta$ свидетельствует об обратном.

На рис. 4 и рис. 5 изображена часть поперечного сечения образца с надрезом, где линией $1_{\mathrm{o}}$ показана величина номинальных напряжений, а линией 2 к - действительные напряжения по контуру дна концентратора. 
С целью создания формулы для инженерного расчета локального теоретического коэффициента концентрации напряжений предлагается связать $\alpha_{\sigma}\left(\Theta_{i}\right)$ и $\alpha_{\sigma}\left(0^{o}\right)=\alpha_{\sigma}$ для детали с угловой координатой $\Theta$ следующей зависимостью:

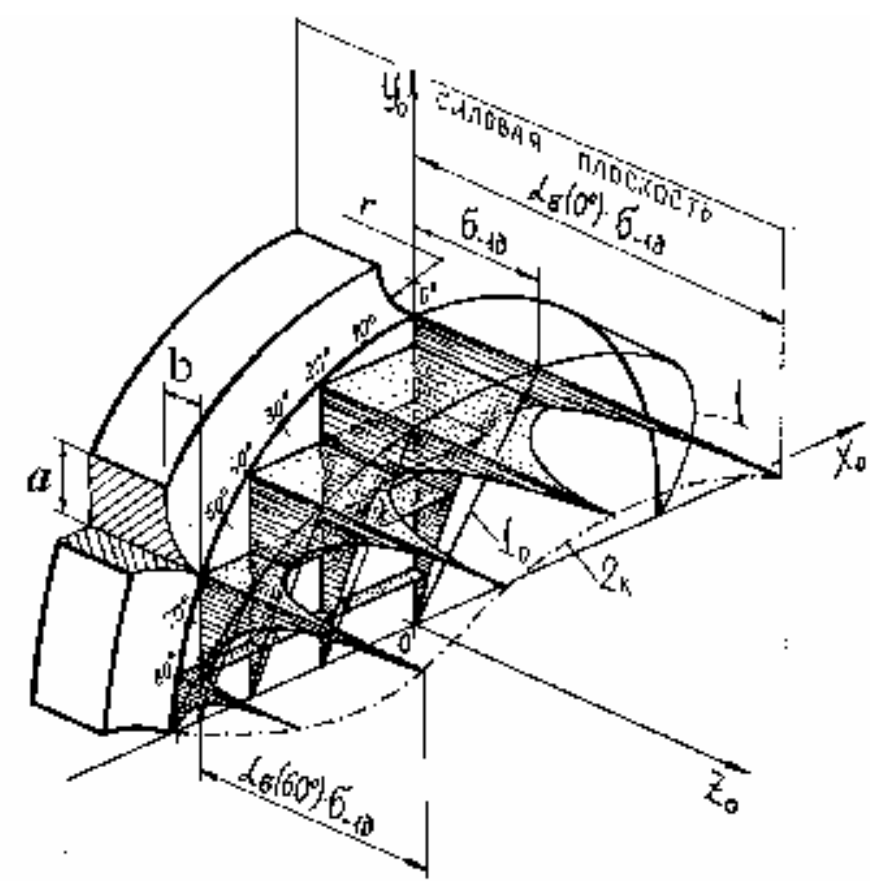

Р и с. 4. Распределение номинальных (плоскость $1_{\mathrm{o}}-1$ ) действительных нормальных напряжений в опасном сечении детали и на поверхности дна надреза (кривая $2_{\text {к) }}$

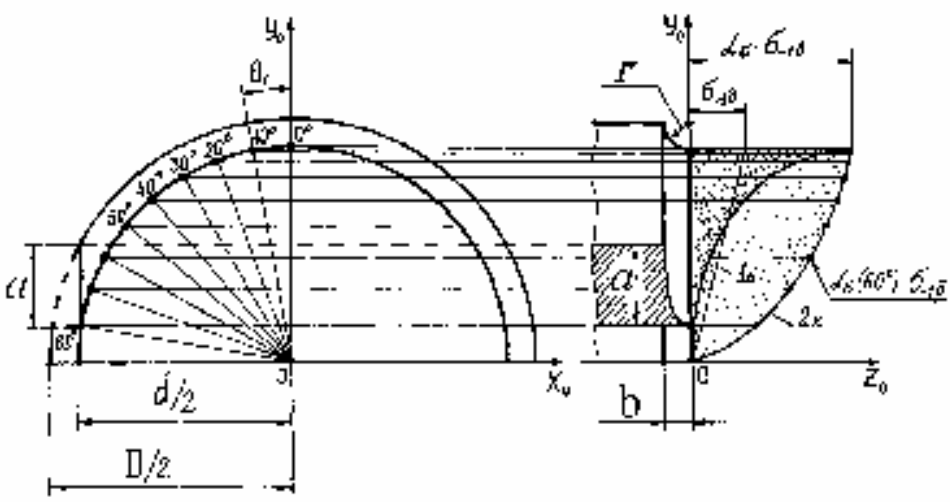

Р и с. 5. Изменение формы полуэллиптического концентратора при сечении вертикальной плоскостью, параллельной силовой, через $10^{\circ}$. Линия $1_{0}$ - эпюра номинальных напряжений в главной (силовой) плоскости жесткости; Линия $2_{\text {к }}$ величина действительных напряжений по контуру дна полукруглого надреза

- многочлен 4-й степени -

$$
\begin{aligned}
& n_{i}=51,639 \cdot \Theta_{i}^{4}-177,9 \cdot \Theta_{i}^{3}+ \\
& 228,91 \cdot \Theta_{i}^{2}-135,65 \cdot \Theta_{i}+34,539 .
\end{aligned}
$$

Графический анализ показал, что наиболее подходящей функцией $n\left(\Theta_{i}\right)$ является многочлен четвертой степени и выше. Таким образом, используя формулы многочлена четвертой степени для показателя корня степени $n\left(\Theta_{i}\right)$ для формулы (11) локального коэффициента концентрации напряжений $\alpha_{\sigma}\left(\Theta_{i}\right)$, можно прогнозировать предел выносливости по первой макротрещине в инженерном приближении, выполняя вычисления по формуле (7). 
Результаты расчетов локального теоретического коэффициента концентрации напряжений $\alpha_{\sigma}\left(\Theta_{i}\right)$ через $10^{\circ}$ центрального угла $\Theta$ и $\alpha_{\sigma}$ по Нейберу

\begin{tabular}{|c|c|c|c|c|c|c|}
\hline$i$ & $\Theta$, град & $\Theta$, рад & $n_{i}$ & $\begin{array}{c}\alpha_{\sigma}\left(\Theta_{i}\right) \text { фор- } \\
\text { мула(11) }\end{array}$ & $\begin{array}{c}\alpha_{\sigma}\left(\Theta_{i}\right) \text { по Ней- } \\
\text { беру }\end{array}$ & $\Delta, \%$ \\
\hline \hline 1 & 10 & $\pi / 18$ & 17 & 3,028 & 3,028 & 0 \\
\hline 2 & 20 & $\pi / 9$ & 8 & 3,112 & 3,117 & 0,16 \\
\hline 3 & 30 & $\pi / 6$ & 5 & 3,250 & 3,280 & 0,9 \\
\hline 4 & 40 & $2 \pi / 9$ & 3 & 3,540 & 3,540 & 0 \\
\hline 5 & 50 & $5 \pi / 18$ & 2 & 3,987 & 3,952 & 0,88 \\
\hline 6 & 60 & $\pi / 3$ & 1,5 & 4,547 & 4,608 & 1,32 \\
\hline 7 & 70 & $7 \pi / 18$ & 1,05 & 5,638 & 5,700 & 1,1 \\
\hline
\end{tabular}

\section{2. Математический анализ}

2.1. Критерий безопасного повреждения детали. Для математического анализа геометрии излома исследуемой детали требуется графическая обработка фотографий (рис.1 и 2) в виде чертежей с копированием изображения границ серповидной усталостной трещины, эффективной области перехода к магистральной трещине и обозначением необходимых угловых и линейных размеров. На рис.6 изображена симметричная половина опасного сечения исследуемой детали. Измерения на исследуемом реальном сечении показали: $2 \Theta_{\max }=120^{\circ}$, $2 \Theta_{э \phi}=60^{\circ}, t_{\max }=1,2 \mathrm{MM}$.

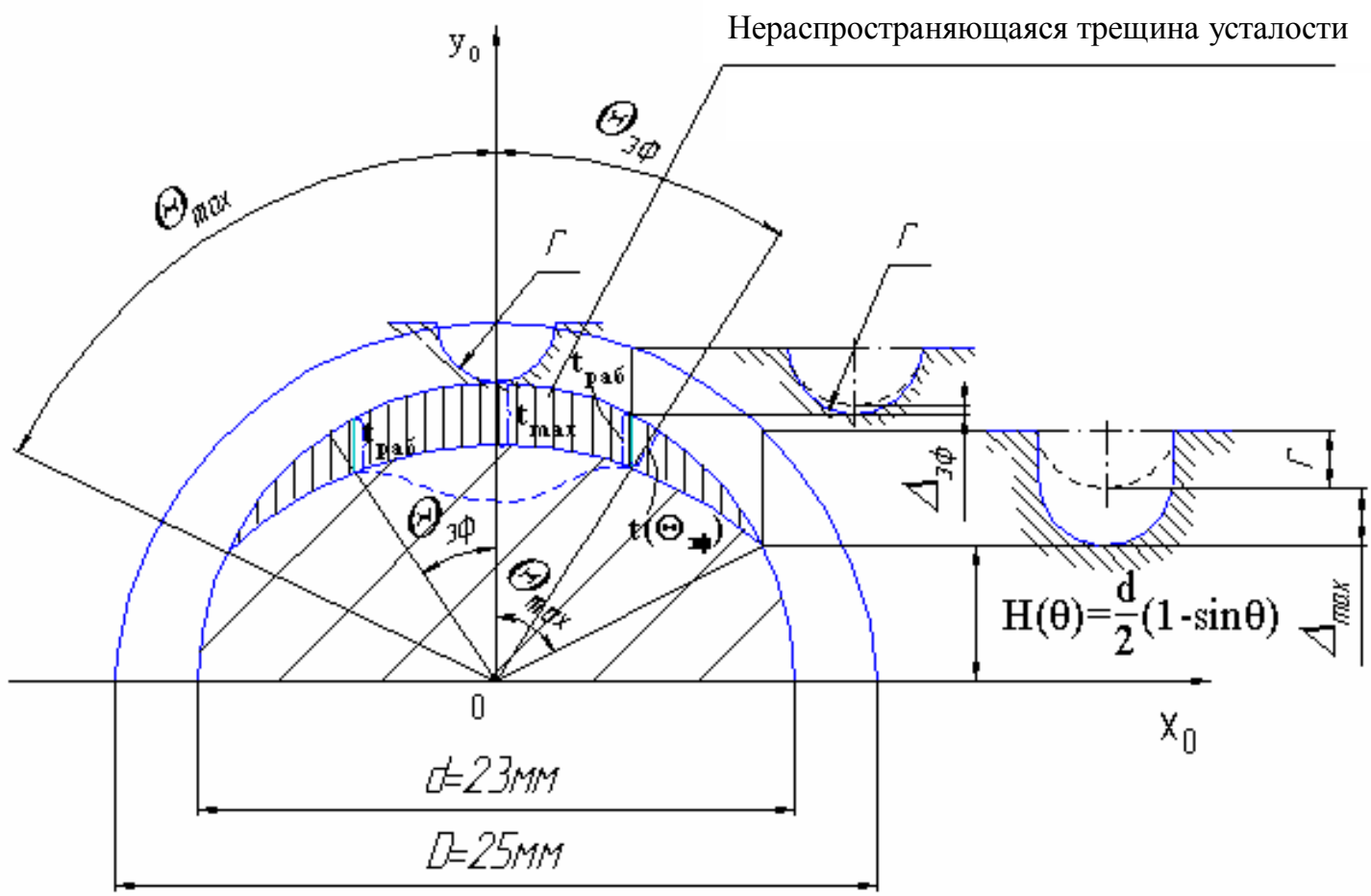

Р и с. 6.Усталостный излом детали с нераспространяющейся трещиной усталости, характерный для стальных сплавов

Нераспространяющаяся трещина усталости серповидной формы, изображенная на рис.6, представляет собой критическое состояние, после которого наступает развитие магистральной трещины в пределах удвоенного эффективного центрального угла $\Theta_{э \phi}$. Является ли в этом случае $t_{\max }$ контрольной глубиной, до которой деталь с трещиной может эксплуатироваться? 
Как правило, контрольная глубина усталостной трещины рассчитывается с помощью коэффициента запаса (или коэффициента размаха трещины), который на практике устанавливается в пределах $n_{\text {тр }}=2 \ldots 3$ [1]. Каким же образом можно рассчитать контрольную глубину усталостной трещины, которую можно также назвать безопасной глубиной усталостной трещины или критерием безопасного повреждения детали, учитывающим физические свойства материала детали?

Предлагается рассматривать максимальную глубину нераспространяющейся усталостной трещины $t_{\max }$ как произведение эффективного коэффициента концентрации напряжений детали $\mathrm{K}_{\sigma}$ и какой-либо условной глубины, рассматривая которую в пределах центрального угла $2 \Theta_{э \phi}$, можно назвать эффективной, т.е. $t_{э \phi}$ :

$$
t_{\max }=\mathrm{K}_{\sigma} \cdot t_{э \phi} \cdot
$$

Однако, если мы рассматриваем центральный угол $2 \Theta_{э ф}$, то $t_{э \phi}$ может представлять собой среднее центрального распределения между $t_{\max }$ и двумя рабочими глубинами, т.е. $2 t_{\text {раб }}$, представляющими собой размер трещины по вертикальной линии, параллельной главной оси OY, вдоль которой определяется $t_{\max }$. При этом $t_{\text {раб }}$ слева от оси ОY, т.е. при $+\Theta$, равна $t_{\text {раб }}$ справа от оси OY, т.е. при - $\Theta$. Тогда коэффициент $\mathrm{K}_{\sigma}$ должен зависеть от текущей угловой координаты $\Theta$. При делении центрального угла $\Theta_{э ф}$ на какое-то количество равных углов $\Delta \Theta$, так называемая эффективная глубина усталостной трещины $t_{э \phi}$, в соответствии с рис.6, выражается следующей формулой:

$$
t_{\ni \phi}\left(\Theta_{i}\right)=\frac{t_{\max }+2 \cdot t_{p a \hat{\sigma}}\left(\Theta_{i}\right)}{3} \cdot \frac{1}{\mathrm{~K}_{\sigma}\left(\Theta_{i}\right)},
$$

где

$$
t_{p a \tilde{\sigma}}\left(\Theta_{i}\right)=t_{\max }\left(1-\frac{\operatorname{tg} \Theta_{i}}{\operatorname{tg} \Theta_{\max }}\right)
$$

- рабочая глубина усталостной трещины при изгибе;

$$
\mathrm{K}_{\sigma}\left(\Theta_{i}\right)=\mathrm{K}_{\sigma} \cdot\left(1+\sin \Theta_{i}\right)
$$

- эффективный коэффициент концентрации напряжений, зависящий от текущей угловой координаты $\Theta_{i}$.

Формула (15) применяется для математического моделирования (в первом приближении) с целью предварительной оценки расчетных результатов. Здесь эффективный коэффициент концентрации напряжений $\mathrm{K}_{\sigma}$ определяется по известной формуле через теоретический коэффициент концентрации напряжений $\alpha_{\sigma}$ детали и коэффициент чувствительности материала $\mathrm{q}_{\sigma}$. В тоже время, для более детального исследования $\mathrm{K}_{\sigma}\left(\Theta_{i}\right)$ целесообразно вычислять с использованием локального теоретического коэффициента концентрации напряжений $\alpha_{\sigma}\left(\Theta_{i}\right)$ (формула (11)) и $q_{\sigma}$. Для этого необходимо специальное широкомасштабное рассмотрение данного вопроса, который выходит за рамки настоящей работы.

Если при $\Theta_{i}$ учесть абсолютный размер $2 \mathrm{H}\left(\Theta_{i}\right)$ между соответствующими симметричными относительно оси ОХ точками на линиях фронтов двух усталостных трещин в виде коэффициента $\mathrm{K}_{d}$, то формула (13) с учетом (14) и (15) примет следующий вид:

$$
t_{\ni \phi}\left(\Theta_{i}\right)=\frac{t_{\max }}{3} \cdot \frac{\left[1+2 \cdot\left(1-\frac{\operatorname{tg} \Theta_{i}}{\operatorname{tg} \Theta_{\max }}\right)\right]}{\mathrm{K}_{\sigma} \cdot\left(1+\sin \Theta_{i}\right)} \cdot \mathrm{K}_{d}\left(2 \mathrm{H}\left(\Theta_{i}\right)\right),
$$

где

$$
\mathrm{H}\left(\Theta_{i}\right)=\frac{d}{2}-t_{\max } \cdot\left(\cos \Theta_{i}-\frac{\sin \Theta_{i}}{\operatorname{tg} \Theta_{\max }}\right) .
$$


Для вычисления $t_{\text {эф}}\left(\Theta_{i}\right)$ при изменении угла $\Theta_{i}$ в пределах $0^{0} \leq \Theta_{i} \leq 30^{0}$ следует разбить угол $\Theta_{э \phi}$ на $n$ равных углов и определить эффективную величину $t_{э \phi}\left(\Theta_{i}\right)$. В табл. 2 представлены результаты вычисления $t_{\ni \phi}\left(\Theta_{\mathrm{i}}\right)$ при $\mathrm{K}_{\sigma}=1,58 ; \alpha_{\sigma}=2,03$ (по результатам расчетов в ANSYS).

Т а бл и ца 2

\begin{tabular}{|c|c|c|c|c|c|c|}
\hline $\mathrm{i}$ & $\Theta_{i}$ (град) & $t\left(\Theta_{i}\right)$ & $t_{\text {раб }}$ & $2 \cdot \mathrm{H}\left(\Theta_{i}\right)$ & $\mathrm{K}_{d}\left(2 \cdot \mathrm{H}\left(\Theta_{i}\right)\right)$ & $t_{\text {э }}\left(\Theta_{i}\right)$ \\
\hline 1 & 0 & 1.2000 & 1.2000 & 20.6000 & 0.8763 & 0.6259 \\
\hline 2 & 10 & 1.0615 & 1.0778 & 20.5599 & 0.8766 & 0.4973 \\
\hline 3 & 20 & 0.8907 & 0.9478 & 19.9390 & 0.8820 & 0.4037 \\
\hline 4 & 30 & 0.6928 & 0.8000 & 18.7186 & 0.8924 & 0.3305 \\
\hline
\end{tabular}

На основании данных табл. 2 теперь можно вычислить критерий безопасного повреждения детали, т.е. глубину нераспространяющейся усталостной трещины, при наличии которой деталь может выработать гарантийный ресурс, заданный производителем. Формула критерия безопасного повреждения детали имеет следующий вид:

$$
t_{\text {безоп }}=\frac{1}{n} \cdot \sum_{i=1}^{n} t_{\text {э }}\left(\Theta_{i}\right)=\frac{1}{n} \cdot \sum_{i=1}^{n} \frac{t_{\max }}{3}\left[1+2 \cdot\left(1-\frac{\operatorname{tg} \Theta_{i}}{\operatorname{tg} \Theta_{\max }}\right)\right] \frac{\mathrm{K}_{d}\left(2 \mathrm{H}\left(\Theta_{i}\right)\right)}{\mathrm{K}_{\sigma} \cdot\left(1+\sin \Theta_{i}\right)} .
$$

В соответствии с формулой (18) и данными табл. 2 рассчитаем безопасную глубину усталостной трещины для исследуемой детали из стали 45:

$$
t_{\text {безо }}=\frac{1}{n} \cdot \sum_{i=1}^{n} t_{э ф}\left(\Theta_{i}\right)=\frac{1}{4} \cdot(0,6259+0,4973+0,4037+0,3305)=0,4643(\text { мм }) .
$$

Используя критерий безопасного повреждения детали, теперь можно расчетным путем определить коэффициент размаха трещины [1]:

$$
n_{T P}=\frac{t_{\max }}{t_{\text {безоn }}}=\frac{1,2}{0,4643}=2,58 .
$$

Таким образом, математический анализ усталостного излома детали с трещиной приводит к серьезным результатам исследования.

2.2. Математическое описание фронта усталостной трещины. Так как при переменном изгибе возникает две симметричных относительно нейтральной оси усталостных трещины, можно предположить, что линии фронтов связаны между собой уравнением эллипса. Однако попытки связать координаты экспериментальных точек на линиях фронтов трещин через 5 градусов центрального угла с уравнением эллипса не дали положительного результата. Поэтому предпринята попытка описания линии фронта усталостной трещины с помощью уравнения окружности. Для этого вычислена координата е центра окружности $\mathrm{O}_{1}$, на которой лежат точки 1 , 2, 3 (рис.7).

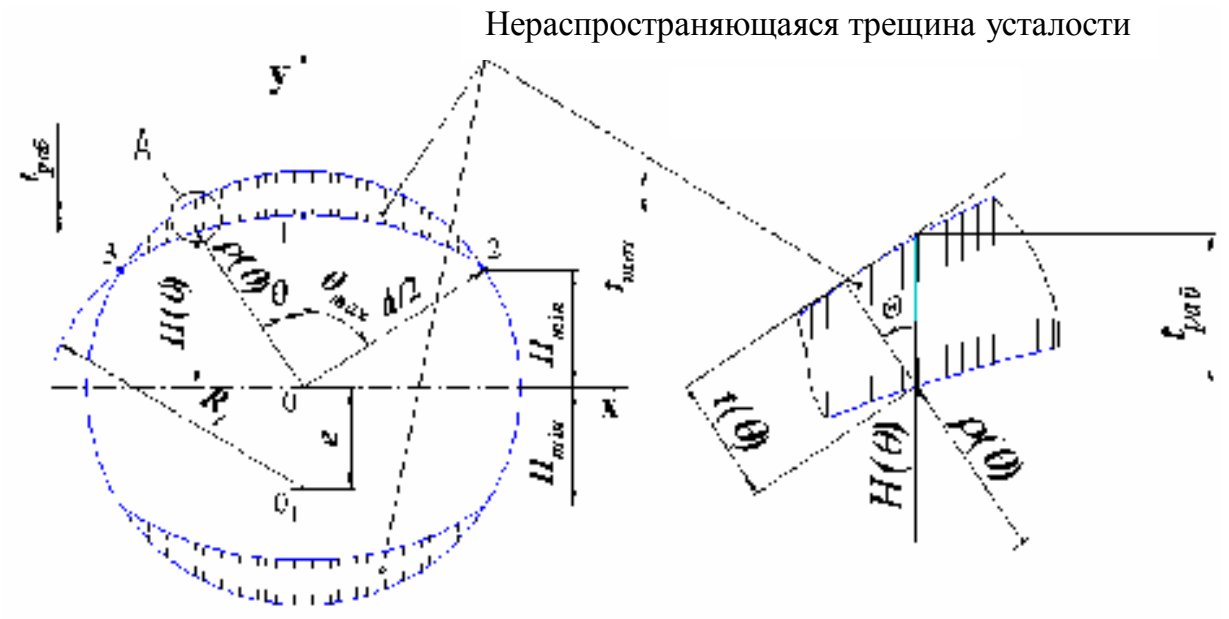

Р и с. 7. Описание фронта усталостной трещины с помощью уравнения окружности 
Уравнение окружности можно получить из условия, $\Theta_{\max }=60^{\circ}$, т.е. окружность проходит через точку 1 с координатами $\left(0 ; \frac{d}{2}-t_{\max }\right)$, точку $2\left(+\frac{\sqrt{3} \cdot d}{4} ; \frac{d}{4}\right)$ и точку $3\left(-\frac{\sqrt{3} \cdot d}{4} ; \frac{d}{4}\right)$. В этом случае уравнение окружности имеет вид:

$$
x^{2}+(y-e)^{2}=R_{t}^{2},
$$

где $e=\left(\frac{d}{2}-t_{\max }-R_{t}\right)$ - величина смещения центра окружности $\mathrm{O}_{1}, R_{t}$ - радиус окружности. Радиус окружности вычисляется по следующей формуле:

$$
R_{t}=\frac{\frac{d}{2} \cdot t_{\max }-\frac{d^{2}}{4}-t_{\max }^{2}}{2 \cdot t_{\max }-\frac{d}{2}}
$$

Переведем уравнение окружности (21) в полярную систему координат с центром в точке $\mathrm{O}$, т.е. в начало координат ХОY, воспользовавшись формулами перевода: $x=\rho \sin \Theta, y=\rho \cos \Theta$. Заменив переменные в уравнении (21), получим квадратное уравнение относительно расстояния от центра окружности О до точек дуги, определяемых $\rho(\Theta)$ :

$$
\rho^{2}(\Theta) \cdot \sin ^{2} \Theta+(\rho(\Theta) \cdot \cos \Theta-e)^{2}-R_{t}^{2}=0 .
$$

Разрешая уравнение (23) относительно $\rho(\Theta)$, получаем уравнение окружности в полярной системе координат:

$$
\rho(\Theta)=e \cos \Theta+\sqrt{R_{t}^{2}-e^{2} \sin ^{2} \Theta} .
$$

С учетом формулы (24) описанные в п.2.1 параметры математической модели примут следующий вид:

$$
\begin{gathered}
t\left(\Theta_{i}\right)=\frac{d}{2}-\rho\left(\Theta_{i}\right) ; \\
t_{p a \sigma}=\frac{\left(\frac{d}{2}-\rho\left(\Theta_{i}\right)\right) \cdot\left(\frac{d}{2}-t_{\max }\right)}{\rho\left(\Theta_{i}\right)} ; \\
t_{э \phi}\left(\Theta_{i}\right)=\frac{1}{6}\left[2 t_{\max }+\left(d-2 \rho\left(\Theta_{i}\right)\right) \cdot\left(d-2 t_{\max }\right)\right] \cdot \frac{K_{d}\left(2 \cdot H\left(\Theta_{i}\right)\right)}{K_{\sigma}\left(1+\sin \Theta_{i}\right)} .
\end{gathered}
$$

В табл. 3 представлены результаты вычислений необходимых параметров при расчетных значениях $R_{t}=13,175 \mathrm{Mм}$ и $e=-2,875 \mathrm{Mм}$.

Т а б ли и ц 3

\begin{tabular}{|c|c|c|c|c|c|c|c|}
\hline $\mathrm{i}$ & $\Theta_{i}$ (град) & $\rho\left(\Theta_{i}\right)$ & $t\left(\Theta_{i}\right)$ & $t_{p a б}$ & $2 \cdot \mathrm{H}\left(\Theta_{i}\right)$ & $\mathrm{K}_{d}\left(2 \cdot \mathrm{H}\left(\Theta_{i}\right)\right)$ & $t_{\text {эq}}\left(\Theta_{i}\right)$ \\
\hline 1 & 0 & 10,3000 & 1.2000 & 1.2000 & 20.6000 & 0.8763 & 0.6259 \\
\hline 2 & 10 & 10,3342 & 1.1658 & 1.0778 & 20.3544 & 0.8784 & 0.5233 \\
\hline 3 & 20 & 10,4366 & 1,0634 & 0.9478 & 19.6145 & 0.8848 & 0.4315 \\
\hline 4 & 30 & 10,6065 & 0.6928 & 0.8935 & 18.3710 & 0.8953 & 0.3476 \\
\hline
\end{tabular}

Исходя из данных табл. 3, глубина безопасного повреждения детали, в соответствии с формулой $(18), t_{\text {безоn }}=0,4821$ мм. Сравнивая с $t_{\text {безоn }}=0,4643$ мм в п.2.1, видим высокую коррелированность результатов. Разница составляет $3,7 \%$, что свидетельствует о высо- кой точности математического моделирования сечения излома детали.

\section{3. Синтез}

3.1. Концентрация напряжений в окрестности фронта усталостной трещины. Анализ линии фронта трещины с помощью оптического микроскопа свидетельствует о том, что ее толщина определяется зоной пластических деформаций $[8,9,15]$. В случае медленного роста усталостной трещины, а при эксплуатационных напряжениях - ниже допускаемой предельной величины, рост трещины практически отсутствует, макродеформации в области фронта трещины считаются упругими $[11,12]$, т.е. микропластические деформации оказывают влияние толь- 
ко на инкубационный период дальнейшего роста трещины [15]. Следовательно, при визуальной регистрации максимальной глубины трещины в главной (силовой) плоскости жесткости $\mathrm{t}_{\max }$ ее расчет целесообразно выполнять путем вычета толщины зоны пластических деформаций, которая легко регистрируется оптическим микроскопом, а также рассчитывается по формуле Губера-Мизеса-Генки $[13,14]$. В нашем случае экспериментальное значение $\mathrm{t}_{\max }$ составляет 1,2мм. Как видно из рис.8, фронт усталостной трещины представляет собой гладкую кривую линию, описываемую уравнением окружности радиусом $\mathrm{R}_{\mathrm{t}}$.

Исходя из вышеизложенного, при изгибе детали линию фронта нераспространяющейся усталостной трещины можно считать линией равных напряжений [10]. В таком случае при выходе линии фронта усталостной трещины на поверхность дна кольцевого надреза опасного сечения детали в точке В действительные нормальные напряжения в локальной плоскости, параллельной главной (силовой) плоскости жесткости YOZ, должны быть равны действительным напряжениям на дне (на линии фронта) усталостной трещины, что записывается в следующем виде:

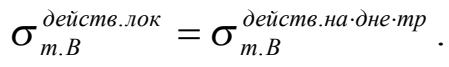

На рис. 8 изображена четвертая часть симметричного поперечного сечения излома (рис.1) с методическим указанием необходимых линейных размеров и действительных напряжений в точке $\mathrm{B}$, определяемой центральным углом (для сталей) $\Theta_{\max }=60^{\circ}$. Параметры нераспространяющейся усталостной трещины полуэллиптической формы - большая полуось и малая полуось - обозначаются соответственно $a_{T P}$ и $b_{T P}$.

Если в выражении (28) соответствующие действительные напряжения расписать как произведение локального теоретического коэффициента концентрации напряжений и номинальных амплитудных напряжений, то полученное соотношение (29), в соответствии с линейной механикой разрушения, будет являться законообразующей формулой №1:

$$
\alpha_{\sigma}^{\text {лок }}\left(\Theta_{\max }\right) \cdot \sigma_{\text {ном }}\left(\Theta_{\max }\right)=\alpha_{\sigma н}^{a . \partial н е т р . ~}\left(\Theta_{i}\right) \cdot \sigma_{\text {ном }}^{\text {нам уровнеднатр. }}\left(\Theta_{i}\right),
$$

где

$$
\sigma_{\text {ном }}\left(\Theta_{\max }\right)=\frac{2 \mathrm{H}_{\min }}{d} \cdot \sigma_{R a}=\frac{2 R \cdot \sin \Theta_{\max }}{d} \cdot \sigma_{R a}
$$

- номинальные напряжения на дне кольцевого надреза в точке выхода линии фронта трещины на поверхность в точке В;

$$
\sigma_{\text {ном }}^{\text {насуровне } \text { дна } \cdot m р .}\left(\Theta_{i}\right)=\frac{\rho\left(\Theta_{i}\right) \cdot \cos \Theta_{i}}{\frac{d}{2}-t_{\max }} \cdot\left(1-\frac{2 t_{\max }}{d}\right) \sigma_{R a}
$$

- номинальные напряжения на уровне любой точки линии дна нераспространяющейся усталостной трещины, $R=d / 2-$ радиус опасного сечения, $\rho\left(\Theta_{i}\right)$ - полярный радиус-вектор,

$$
\begin{gathered}
\mathrm{H}_{\text {min }}=R \cdot \sin \Theta_{\max } ; \\
\mathrm{H}\left(\Theta_{i}\right)=\rho\left(\Theta_{i}\right) \cdot \cos \Theta_{i} .
\end{gathered}
$$

Ведение полярных координат позволяет определять положение любой точки на линии фронта усталостной трещины посредством радиус-вектора $\rho$ и центрального угла $\Theta_{i}$, отсчитываемые от точки О в начале декартовой системы координат. С другой стороны линия фронта трещины также описывается дугой окружности радиусом $\mathrm{R}_{\mathrm{t}}$ с точкой отсчета $\mathrm{O}_{1}$, смещенной по вертикали относительно точки О на величину е. Выражения $\rho\left(\Theta_{i}\right), \mathrm{R}_{\mathrm{t}}, e$ представлены следующими формулами:

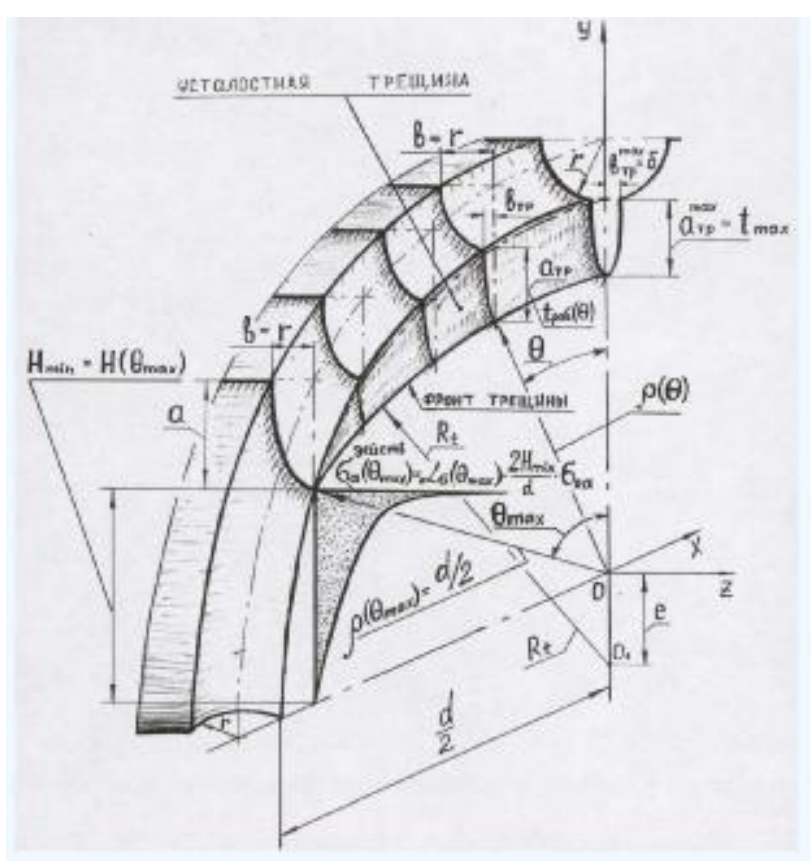

Р и с. 8. Пересечение фронта нераспространяющейся усталостной трещины с контуром дна надреза 


$$
\begin{gathered}
\rho\left(\Theta_{i}\right)=e \cdot \cos \Theta_{i}+\sqrt{R_{t}^{2}-e^{2} \cdot \sin ^{2} \Theta_{i}}, \\
R_{t}=\frac{\frac{d}{2} \cdot t_{\max }-\frac{d^{2}}{4} \cdot t_{\max }^{2}}{2 \cdot t_{\max }-\frac{d}{2}}, \\
e=\frac{d}{2}-t_{\text {max }}-R_{t} .
\end{gathered}
$$

Из выражения (29) и составляющих его номинальных напряжений на дне надреза в точке В и на уровне дна трещины выведем формулу теоретического коэффициента концентрации напряжений для любой точки на линии (в окрестности) фронта усталостной трещины, которая имеет следующий вид:

$$
\alpha_{\sigma}^{\text {на.уровне.дна.ТР. }}\left(\Theta_{i}\right)=\alpha_{\sigma}^{\text {лок }}\left(\Theta_{\max }\right) \cdot \frac{\sigma_{\text {ном }}\left(\Theta_{\max }\right)}{\sigma_{\text {но.м }}^{\text {наввне.дна.ТР. }}\left(\Theta_{i}\right)} .
$$

Исходя из формулы (37), следует вывод о том, что концентрация напряжений в окрестности фронта трещины будет меньше концентрации напряжений на поверхности дна надреза по линии вертикального сечения, параллельного силовой плоскости. Этот вывод совпадает с исследованиями в работах $[15,16,17]$. При этом необходимо иметь ввиду, что при $\Theta_{\max }$, т.е. в точке $\mathrm{B}$, локальный теоретический коэффициент концентрации напряжений и номинальные напряжения являются постоянными величинами.

Сама по себе линия фронта нераспространяющейся усталостной трещины представляет собой область равновесия действия внешней нагрузки и противодействующих внутренних напряжений $[16,17]$. При переменном изгибе, когда напряжения больше предела выносливости детали по первой макротрещине, в поперечном сечении возникают две симметричные усталостные трещины. Расстояние между двумя симметричными точками на соответствующих линиях фронтов трещин изменяется от $2 \mathrm{H}\left(0^{o}\right)=\left(d-2 \cdot t_{\max }\right)$ в силовой плоскости до $2 \mathrm{H}_{\min }=d \cdot \cos \Theta_{\max }$ в произвольной плоскости, параллельной силовой. Возникает вопрос: возможна ли связь между линейными размерами усталостного излома и его физическими параметрами, в частности, напряжениями? Ответ на этот вопрос целесообразно искать в относительных величинах. Для этого необходимо выполнить вычисления, в соответствии с формулами (11) и (29)-(37), а также вычислить разницу теоретических коэффициентов концентрации напряжений на дне надреза и на дне трещины $\Delta \alpha_{\sigma}$ по линии вертикали, опущенной из точки пересечения луча, проведенного через $10^{\circ}$ из начала координат (точке $\mathrm{O}$ на рис.8) до пересечения с окружностью линии дна опасного сечения трещины. Результаты вычислений представлены в табл. 4. Следует иметь ввиду то, что $\Theta_{0}=0^{0}$ определяет главную (силовую) плоскость жесткости.

Из табл. 4 видно, что относительные величины в строках 7 и 8 абсолютно одинаковы. Это позволяет установить алгебраическое равенство данных соотношений, которое можно представить в следующем виде:

где

$$
\alpha_{\sigma}^{m p .}\left(\Theta_{0}\right) \cdot \mathrm{H}\left(\Theta_{0}\right)=\alpha_{\sigma}^{m p} \cdot\left(\Theta_{i}\right) \cdot \mathrm{H}\left(\Theta_{i}\right)
$$

$$
\mathrm{H}\left(\Theta_{0}\right)=\frac{d}{2}-t_{\max } .
$$

Как видно, выражение (38) представляет собой законообразующую формулу №2. Удвоенная величина $\mathrm{H}\left(\Theta_{0}\right)$ есть ни что иное, как расстояние между двумя серповидными усталостными трещинами в главной плоскости жесткости, т.е. в силовой плоскости. Таким образом, формула (38) определяет связь геометрических параметров трещинообразования и физических параметров опасного сечения с конструктивным концентратором, в частности, теоретического коэффициента концентрации напряжений в области дна трещины.

Подставляя (39) в (38) и выделяя $t_{\max }$, получаем теоретическую формулу расчета максимальной глубины усталостной трещины в силовой плоскости: 


$$
t_{\max }=\frac{d}{2}-\mathrm{H}\left(\Theta_{i}\right) \cdot \frac{\alpha_{\sigma}^{m p}\left(\Theta_{i}\right)}{\alpha_{\sigma}^{m p}\left(0^{0}\right)} .
$$

Т а б ли и а 4

\begin{tabular}{|c|c|c|c|c|c|c|c|c|}
\hline$\stackrel{201}{z}$ & & $0^{\circ}$ & $10^{\circ}$ & $20^{\circ}$ & $30^{\circ}$ & $40^{\circ}$ & $50^{\circ}$ & $60^{\circ}$ \\
\hline 1 & $\begin{array}{c}\rho(\Theta), \\
\text { MM }\end{array}$ & 10,30 & 10,34 & 10,44 & 10,60 & 10,84 & 11,14 & 11,50 \\
\hline 2 & $\begin{array}{r}\mathrm{H}(\Theta), \\
\mathrm{MM} \\
\end{array}$ & 10,30 & 10,18 & 9,81 & 9,18 & 8,30 & 7,16 & 5,75 \\
\hline 3 & $\begin{array}{c}\sigma_{\text {ном }}^{m p}(H(\Theta) \\
\text { МПа }\end{array}$ & 172,2 & 170,20 & 164,0 & 153,50 & 138,8 & 119,7 & 96,15 \\
\hline 4 & $\alpha_{\sigma}^{\text {лок }}(\Theta)$ & 3,00 & 3,028 & 3,117 & 3,280 & 3,540 & 3,990 & 4,600 \\
\hline 5 & $\alpha_{\sigma}^{m p}(\Theta)$ & 2,57 & 2,60 & 2,696 & 2,880 & 3,187 & 3,690 & 4,600 \\
\hline 6 & $\Delta \alpha_{\sigma}$ & 0,43 & 0,429 & 0,421 & 0,400 & 0,353 & 0,300 & 0,000 \\
\hline 7 & $\frac{\mathrm{H}\left(\Theta_{0}\right)}{\mathrm{H}(\Theta)}$ & 1,009 & 1,012 & 1,050 & 1,120 & 1,240 & 1,440 & 1,790 \\
\hline 8 & $\frac{\alpha_{\sigma}^{m p}(\Theta)}{\alpha_{\sigma}^{m p}\left(\Theta_{0}\right)}$ & 1,01 & 1,012 & 1,049 & 1,120 & 1,240 & 1,440 & 1,790 \\
\hline
\end{tabular}

Используя данные табл. 4 и приняв для расчета необходимые данные, например при $\Theta=30^{\circ}$,вычислим максимальную глубину серповидной нераспространяющейся усталостной трещины по формуле (40):

$$
t_{\max }=\frac{23,0}{2}-9,18 \cdot \frac{2,88}{2,57}=1,21 \mathrm{MM} .
$$

Результат экспериментального замера максимальной глубины усталостной трещины за вычетом толщины пластической зоны, который отмечен в ранних публикациях автора при $t_{\max }=1,20$ мм , свидетельствует об идеальной сходимости теоретической концепции физикоматематического анализа усталостного излома детали с измерениями реального сечения статического излома исследуемой детали с усталостной трещиной.

При исследовании трещинообразования проводится прочностной анализ, основанный на расчетах коэффициента интенсивности напряжений (КИН) в соответствии с теорией Гриффитса-Ирвина. Однако здесь имеет место вопрос, на который ученые еще не дали ответ: почему КИН при остановке трещины меньше КИН при ее последующем страгивании, ведь прирост длины трещины в этот момент равен нулю? Очевидно, снимать с повестки дня концентрацию макронапряжений в окрестности дна трещины рано, несмотря на то, что на ее дне имеют место микропластические деформации. Ответ на этот вопрос целесообразно искать, рассматривая поведение материала за пределом текучести.

Известно, что за пределом текучести при разгрузке и последующей нагрузке материал испытывает упругие деформации. В этом случае номинальные рабочие напряжения детали могут быть выше предела пропорциональности материала. Следовательно, микропластические деформации на дне трещины, способствуя переходу дислокаций из одной формы в другую[18], приводят к тому, что процесс продвижения трещины возможен при вторичной стадии пластичности - квазипластичности, возникающей при напряжениях больше предела прочности материала, определяющих вязкость материала.

Если проанализировать изменение величины действительных напряжений по контуру опасного сечения исследуемой цилиндрической детали с использованием локального теоретического коэффициента концентрации напряжений, то расчеты свидетельствуют о том, что в 
пределах эффективного центрального угла относительно главной плоскости жесткости (силовой плоскости) действительные напряжения изменяются всего лишь на $2,5 \%$. В тоже время, в точке выхода серповидной трещины на поверхность дна конструктивного кольцевого надреза действительные напряжения относительно напряжений в силовой плоскости отличаются примерно на 35\%. Этот факт объясняет мгновенное распространение усталостной трещины в пределах от эффективного центрального угла до максимального при проведении усталостных испытаний с целью определения предела выносливости по первой макротрещине в случае изгиба. Так как магистральное развитие усталостной трещины происходит в пределах удвоенного эффективного центрального угла, то изучение развития трещины в пределах так называемого наплыва (рис. 2) заслуживает большого внимания в будущих научных исследованиях.

Таким образом, физико-математический анализ усталостного излома детали свидетельствует о том, что вопросы концентрации макронапряжений в окрестности фронта усталостной трещины нельзя исключать из рассмотрения, основываясь только на расчетах коэффициента интенсивности напряжений по теории Гриффитса-Ирвина и изучении микронапряжений в зонах разрушения деталей. Представленная методика физико-математического анализа излома детали является неотъемлемой частью прочностного анализа работоспособности не только детали, но и конструкции в целом. Она позволяет установить связь между геометрическими параметрами нераспространяющейся трещины и ее физическими характеристиками в окрестности линии фронта, а также между параметрами трещинообразования и параметрами усталостного разрушения детали в условиях концентрации напряжений. Как видно, концентрация макронапряжений в окрестности линии фронта усталостной трещины обладает собственной механикой, требующей развития в научно-исследовательской работе.

Предлагаемая методика физико-математического анализа усталостного излома деталей на этом не исчерпывается. Она позволяет рассчитывать концентрацию напряжений в опасной точке в момент страгивания трещины, т.е. еще до ее появления. Это позволяет ответить на вопрос: почему коэффициент интенсивности напряжений при страгивании трещины больше коэффициента интенсивности напряжений при ее остановке? Методика позволяет прогнозировать диапазоны безопасной работы детали с трещиной при сложном нагружении, связывает коэффициент интенсивности напряжений с остаточными напряжениями после поверхностного пластического деформирования. Теоретические основы методики обладают относительной простотой и удобством в использовании, что актуально в инженерной практике.

\section{БИБЛИОГРАФИЧЕСКИЙ СПИСОК}

1. Жилюкас А.Ю. Разрушение конструкционных элементов. Вильнюс: Мокслас. 1988. 108с.

2. Кольиун Ю.И., Денискина Е.А., Хибник Т.А. Математическое моделирование эффективной величины нераспространяющейся трещины усталости // Математ. моделирование и краевые задачи. Труды 12 межвуз. конф. Часть 1. Самара: СамГТУ, 2002. С.87-90.

3. Кольиун Ю.И. Определение предела выносливости материала по образованию первой макротрещины в условиях концентрации напряжений // Высокие технологии в машиностроении. Материалы МНТК. Самара: СамГТУ, 2002. C.102-105.

4. Кольиун Ю.И. Локальный теоретический коэффициент концентрации напряжений при прогнозировании предела выносливости по первой макротрещине // Проблемы прочности материалов и сооружений на транспорте. Труды 6 МНТК. Санкт-Петербург: ПГУПС. 2004. С.181-190.

5. Кольиун Ю.И. Прогнозирование предела выносливости по образованию первой макротрещины при изгибе // Вестник СГАУ. Спец. Выпуск / Труды МНТК «Проблемы и перспективы развития двигателестроения», Самаpa: СГАУ, 2003. С.222-226.

6. Кудрявцев П.И., Морозова Т.И. О критическом размере нераспространяющихся усталостных трещин в стальных поверхностно наклепанных деталях // Вопросы прочности крупных деталей машин. М.: Машиностроение, 1976. С.247-256.

7. Кудрявцев П.И. Нераспространяющиеся усталостные трещины. М.: Машиностроение, 1982. 174c.

8. Махутов Н.A. Сопротивление элементов конструкций хрупкому разрушению. М.: Машиностроение, 1973. 201c.

9. Морозов Е.М. Механика разрушения упругопластических тел: Учебное пособие. М.: МИФИ, 1986. $88 \mathrm{c}$.

10. Нейбер Г. Концентрация напряжений. М.- Л.: Гостехиздат. 1947.

11. Партон В.3. Механика разрушения. От теории к практике. М.: Наука, 1990. 238c.

12. П Партон В.3., Морозов Е.М. Механика упругопластического разрушения. М.: Наука, 1985. 504c.

13. Петерсон P.E. Коэффициенты концентрации напряжений. М.: Мир, 1977. 302c.

14. Писаренко Г.С. Справочник по сопротивлению материалов. Киев: Вища школа, 1982.

15. Серенсен С.В. Сопротивление материалов усталостному и хрупкому разрушению. М. Атомиздат, $1975.191 \mathrm{c}$.

16. Слепян Л.И. Механика трещин. Л.: Судостроения, 1981. 296с.

17. Черепанов Г.П. Механика хрупкого разрушения. М.: Наука, 1974г. 640с.

18. Яковлева Т.Ю. Локальная пластическая деформация и усталость металлов. Киев: Наукова Думка, 2003. 236с. 